\title{
Method for estimating the propagation direction of a coherent plasma structure using a one-dimensional diagnostic array
}

\author{
T. Kobayashi ${ }^{1,6}$, G. Birkenmeier ${ }^{2}$, E. Wolfrum ${ }^{2}$, F. M. Laggner ${ }^{3}$,

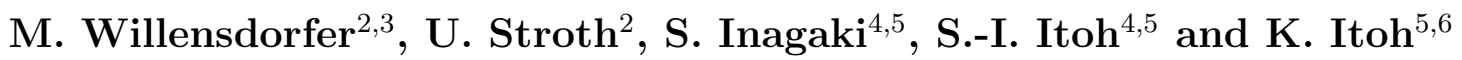 \\ ${ }^{1}$ Interdisciplinary Graduate School of Engineering Sciences, Kyushu University, Kasuga \\ 816-8580, Japan \\ ${ }^{2}$ Max Planck Institute for Plasma Physics, Boltzmannstr. 2, 85748 Garching, Germany \\ ${ }^{3}$ Institute of Applied Physics, Vienna University of Technology, 1040 Vienna, Austria \\ ${ }^{4}$ Research Institute for Applied Mechanics, Kyushu University, Kasuga 816-8580, Japan \\ ${ }^{5}$ Research Center for Plasma Turbulence, Kyushu University, Kasuga 816-8580, Japan \\ ${ }^{6}$ National Institute for Fusion Science, Toki 509-5292, Japan \\ E-mail: kobayashi.tatsuya@LHD.nifs.ac.jp
}

\begin{abstract}
This article proposes a new method to evaluate basic characteristics of the dynamics of a coherent plasma structure (blob). With this method, one can evaluate the propagation angle of a blob in a two-dimensional plasma cross section as well as the blob velocity, size, and amplitude from one-dimensional data. The method is applied to blob measurements from the Lithium beam emission spectroscopy (Li-BES) system in ASDEX-Upgrade (AUG). Statistical features of the observed blob velocities, angles of propagation, blob sizes and amplitudes are discussed. The validity of the method is examined by comparing two values of the propagation angle that are evaluated in an independent manner.
\end{abstract}




\section{Introduction}

The dynamics of coherent plasma structures are intensively studied [1] not only for gaining scientific understanding of this self-organization but also in view of realization of a thermonuclear reactor. For instance, in order to reduce the heat load towards the first wall or divertor of a nuclear reactor, the dynamics of coherent plasma structures in the scrape-off layer (SOL), so called plasma blob filaments, are deeply investigated (see reviews in $[2,3]$ ). The radial non-diffusive transport of coherent plasma structures produce a non-exponentially decaying density tail in the SOL $[4,5]$, which can lead to the increase of impurities and recycling rate on the first wall. In order to explain the radial transport, several theoretical models are proposed $[6,7,8]$. In these theories, the main mechanism which induces $E \times B$ radial transport is considered to be a $\nabla B$ drift driven charge separation in the blob filament. The charge separation is balanced by creating a closed current loop by, for instance, an ion polarization current or sheath current at the limiter or divertor plate. The blob size determines the dominant element of the current loop closure, which results in a blob size dependence of the radial velocity. In that context, many experimental observations of the interrelation between blob size and radial velocity are performed to examine the theoretical models $[9,10,11,12,13]$.

For the study of blob dynamics including such a blob scaling, two-dimensional measurements are widely used $[9,10,11,14,15,16]$. In addition to the blob size and the radial velocity scaling, two-dimensional measurements of the blob velocity are important because the background poloidal flow and flow shear can affect transport 
processes. Also tilted structures carried by the flow can lead to misinterpretation of the time delays in terms of velocity direction and amplitude [17]. Recently, the focus is directed at poloidal momentum transport by blobs, in order to explain shear flow generation at the boundary of the edge/SOL region $[18,19]$.

Although the two-dimensional measurements develop remarkably in modern plasma experimental systems, some difficulties, e.g., signal interpretation or complication of the installation, as well as spatial restriction, still exist. In contrast, simple one-dimensional measurement is routinely used in many devices. In this article, we propose a new method which enables us to evaluate the propagation angle of a blob in the two-dimensional plasma cross section as well as the blob velocity, size and amplitude. The method is applied to blob measurements from the Lithium beam emission spectroscopy (LiBES) system in ASDEX-Upgrade (AUG) [20]. Statistical features of the observed blob velocities, angles of propagation, sizes and amplitudes are discussed. The validity of the method is examined by comparing two values of the propagation angle that are evaluated in an independent manner.

\section{Analysis method}

The schematic view of the one-dimensional blob detection system is shown in Fig. 1. The Cartesian coordinate drawn in Fig. 1 is used to describe the direction of the blob motion on the poloidal cross-section, where the radial and poloidal directions correspond to $x$ and $y$ directions, respectively. For simplicity, the following three assumptions of 
propagation characteristics are used for the explanation: (1) The blob has a circular shape in the poloidal cross section with a monopole density perturbation (e.g., twodimensional Gaussian distribution) $[11,15]$, and keeps its shape in a rigid manner during propagation. (2) The blob propagates with a constant velocity vector. (3) Generation or corruption does not take place on the line of the diagnostic array. The effect of some violations of the assumptions in the practical case is discussed later. The situation that is considered here is that a single blob with a radius $a$ and a velocity $v$ moves through the line of the diagnostic array $l$ with an angle $\theta$. Each detector senses the blob when the distance to the center of the blob ' $\mathrm{O}$ ' is less than $a$. The point ' $\mathrm{C}$ ' represents the intersection between $l$ and the blob trajectory. The points 'A' and 'B' correspond to the intersection between $l$ and the equiphase line of the blob and the projection of ' $O$ ' onto $l$, respectively. From the velocity difference between the points 'A' and 'B', the propagation angle $\theta$ is derived.

The velocity of the point 'A' is identical to that obtained from time delay of the signal peak in the detector array. When 'A' passes the position of the certain detector that we analyze, the signal experiences the maximum in its time evolution. The time when the signal takes the maximum is defined as $t_{\max }(x)$, as a function of the position of the detector $x$. The velocity of 'A' is calculated from the time delay of $t_{\max }(x)$ at different positions of the detectors, i.e., $v_{\text {delay }}=\left[\mathrm{d} t_{\max }(x) / \mathrm{d} x\right]^{-1}$. This is also given as $v_{\text {delay }}=v / \cos \theta$ geometrically. In contrast, the motion of ' $\mathrm{B}$ ' shows the evolution of the peak position on the one-dimensional spatial distribution of the signal. 
The peak position at time $t$ is defined as $x_{\max }(t)$, and the velocity of ' $\mathrm{B}$ ' is given as $v_{\text {peak }} \equiv v_{x}=\mathrm{d} x_{\max }(t) / \mathrm{d} t$. Another expression $v_{\text {peak }}=v \cos \theta$ is also utilized from Fig. 1 (a).

Data processing of a set of signals from the diagnostics gives us $v_{\text {delay }}$ and $v_{\text {peak }}$ simultaneously at every blob event, and from the pair of velocities, the blob velocity $v$ and the propagation direction $\theta$ are obtained as

$$
v=\sqrt{v_{\text {peak }} \times v_{\text {delay }}}
$$

and

$$
|\cos \theta|=\sqrt{v_{\text {peak }} / v_{\text {delay }}}
$$

respectively. In Fig. 1 (b), the illustration which depicts a typical spatiotemporal evolution of a single blob and the trajectory of the points 'A' and 'B' is shown. Short vertical and horizontal pieces at left bottom corner (right top corner) in the figure show the first (last) appearance of the points 'A' and 'B' in the contour plot. As an example, the positions of ' $\mathrm{A}$ ' and ' $\mathrm{B}$ ' at $t=t^{\prime}$, where the situation corresponds to the picture in Fig. 1 (a), are plotted in Fig. 1 (b). The velocity of the point ' $A$ ' is always faster than that of the point ' $\mathrm{B}$ ', i.e., $v_{\text {peak }}<v_{\text {delay }}$, when $\theta \neq 0$. In addition, when ' $\mathrm{O}$ ' is overlapped on $l$, the radius of the blob is evaluated as the half width at half maximum (HWHM) of the spatial distribution of the signal. The position and time where ' $\mathrm{O}$ ' is just on $l$ can be found at the point which has recorded the maximum value of the signal in the set of spatiotemporal data. Furthermore, the maximum value of the signal itself reflects the amplitude of the blob. With this method, only $|\cos \theta|$ is obtained, therefore, the sign 
of $\theta$ cannot be evaluated. This might be one of the limitations of the one-dimensional observation.

In the practical case, the above three assumptions can sometimes be violated. For example, asymmetric blob shapes, e.g., blob that has a strong gradient in the front and a weak gradient in the rear, have been observed in the experiments and numerical simulations $[21,22,23,24]$. In addition, Li-BES signal generally tends to have an asymmetry in the radial direction because of the finite lifetime of the excited electrons as discussed in Ref. [20]. These asymmetric data shapes result in a distortion to the contour map of the spatiotemporal evolution. In the case of the blob that has steep gradient in the front, the center-of-mass of the contour might be shifted toward the bottom left side. Temporal changes in the blob velocity and size also affect the contour map by bending and/or blurring the trajectory. Nevertheless, as far as one can capture the trajectory of the points 'A' and 'B', the propagation velocity and angle are properly obtained from Eqs. (1) and (2), even if the spatiotemporal evolution contour is distorted.

\section{Application of the method}

The method proposed above is applied to data from an L-mode plasma of the discharge \# 28814, in AUG. The time evolution of the plasma parameters is shown in Fig. 2. The experiment is conducted with the following conditions: the toroidal magnetic field of $B=-2.5 \mathrm{~T}$ (negative sign corresponds to the clockwise direction) with lower single null configuration, the plasma current of $I_{\mathrm{p}}=0.8 \mathrm{MA}$, and the safety factor at the 
flux surface enclosing $95 \%$ of the total poloidal flux of $q_{95}=5.17$. The line-integrated electron density is $\bar{n}_{\mathrm{e}} L \sim 4.5 \times 10^{19} \mathrm{~m}^{-2}$. The edge Thomson scattering system [25] provides the electron temperature in the scrape-off layer (SOL) of $T_{\mathrm{e}} \sim 25 \mathrm{eV}$. The analysis is performed for the time interval $t=2.4-2.8 \mathrm{~s}$, which is indicated by two vertical dashed lines in Fig. 2. In this time interval, the plasma is in L-mode, sustained only with Ohmic heating (no auxiliary heating). According to Figs. 2 (a)(c), which show time evolutions of plasma current $I_{\mathrm{p}}$, plasma stored energy $W_{\mathrm{MHD}}$ and line-integrated electron density $\bar{n}_{\mathrm{e}} L$, respectively, the plasma parameters in this time interval are almost constant. Figure $2(\mathrm{~d})$ indicates constant $D_{\alpha}$ in time, showing no large spikes related to ELM activities. Therefore, any plasma activities except for the blob events are considered not to affect the following analysis. For other discharges with different plasma parameters, this method provides us plausible results likewise.

The main diagnostic of this study is the Lithium beam emission spectroscopy (LiBES) system $[26,20]$. The adaptability of the Li-BES system for blob measurement was recently demonstrated in AUG $[12,20]$. An externally injected neutral Li-beam emits Li I line radiation by reactions with plasma particles, from which the electron density can be deduced. The optical head houses more than 20 channels of detectors (elliptic shape with minor axis and major axis of about $5 \mathrm{~mm}$ and $11 \mathrm{~mm}$, respectively), arranged in the direction of the major plasma radius with $6-7 \mathrm{~mm}$ distance between adjacent channels. The Lithium beam is installed about $32 \mathrm{~cm}$ above the outer (low-field side) mid-plane, where the angle between the beam line and the plasma minor radius 
is smaller than 20 degree. In this discharge, the positions of the detected volumes correspond to $\rho_{\mathrm{p}} \sim 0.92-1.1$ in terms of the normalized poloidal flux coordinate, $\rho_{\mathrm{p}} \equiv \sqrt{\left(\Psi-\Psi_{\mathrm{ax}}\right) /\left(\Psi_{\mathrm{sep}}-\Psi_{\mathrm{ax}}\right)}$, where $\Psi_{\mathrm{sep}}$ and $\Psi_{\mathrm{ax}}$ denote the poloidal fluxes at the separatrix and the magnetic axis, respectively. In particular, in the region of $\rho_{\mathrm{p}}>1$, i.e., in the SOL, the emission intensity is proportional to the electron density. In this region, the skewness of the probability distribution function (PDF) of the signal has a positive trend (up to 0.5 ), showing the appearance of positive spikes due to blobs. The time resolution is $5 \mu \mathrm{s}$. The signal to noise ratio is sufficiently high in the low frequency range, whereas it becomes the order of unity at $f>20 \mathrm{kHz}$ for this discharge. In order to extract the signal components of interest for the blob activity, a digital filter with a band-pass regime $1-10 \mathrm{kHz}[27]$ is used. The regime of filter frequency limits the upper and lower observable boundaries of the blob with respect to the velocity-size ratio $v / a$. The validity of the values are discussed later. Furthermore, a spatial filter is used to eliminate small-scale turbulence from the blob pattern. Here, we focus on blobs with $a>1.5 \mathrm{~cm}$, and smaller structures are filtered out.

A conditional averaging technique is applied for each blob event as performed in Ref. [12]. The reference position is set at $\rho_{\mathrm{p}}=1.043$, and a threshold value of $\mu+3 \sigma$ is employed to determine the onset of a blob event, where $\mu$ and $\sigma$ denote the mean value and the standard deviation of the signal at the reference position. During the time interval $t=2.4-2.8 \mathrm{~s}, 28$ independent blob events are selected. Figure 3 (a) shows a typical spatiotemporal evolution of a single blob in the SOL region at $t \sim 2.513 \mathrm{~s}$. The 
contour map shows the perturbation intensity of the $\operatorname{Li}_{2 \mathrm{p}-2 \mathrm{~s}}$ emission, $\delta I \equiv I-\bar{I}$, where $I$ and $\bar{I}$ denote the emission intensity and its mean value, respectively. Definitions of horizontal and vertical axes in Fig. 3 (a) are: $\Delta t=t-t_{i}$ and $x_{\mathrm{s}}=R-R_{\mathrm{s}}$, where $t_{i}$ is the onset time of the $i$-th blob event and $R$ and $R_{\mathrm{s}}$ are major radii on the line of detectors and separatrix position, respectively. The position of the reference channel is $x_{\mathrm{s}} \sim 4 \mathrm{~cm}$. Figures $3(\mathrm{~b})$ and (c) show slices of the contour diagram at specific positions and time points, respectively. In the contour diagram, the trajectory of 'A' and 'B', i.e., $t_{\max }(x)$ and $x_{\max }(t)$, are plotted. Those points are calculated as the center-of-mass (see definition in [12]) of slice curves shown in Figs. 3 (b) and (c). Linear fit of the trajectories provides $v_{\text {delay }}$ and $v_{\text {peak }}$, which bring $v$ and $\theta$ through Eqs. (1) and (2), respectively. Here, the linear fit averages out all temporal and spatial changes of blob trajectories, and gains one representative velocity and angle for the whole propagation through the SOL. The maximum point of the signal is regarded as the position and time point where 'O' overlaps on $l$. A characteristic width of the blob emission profile $\delta x$ is calculated from the time-constant slice of the contour diagram at that time, as the HWHM value. It should be noted that there is a discrepancy between $a$ and $\delta x$ due to the lifetime of the excited state and the velocity of the beam atoms, as discussed in Ref. $[12,20]$. Because of these effects, perturbation in the emission profile caused by a density perturbation that has a certain width tends to be radially elongated from the original density perturbation width. Sensitivity study well resolved relation between $a$ and $\delta x$, as $a \sim 1.5(\delta x-1) \mathrm{cm}$ in the SOL. In addition to $\delta x$, the amplitude of 
the blob in the emission profile, $\delta I$, is also obtained as the maximum value. Linearity between the relative density perturbation $\delta n_{\mathrm{e}} / \bar{n}_{\mathrm{e}}$ and emission perturbation $\delta I / \bar{I}$ was also demonstrated by the sensitivity study in the SOL region. Therefore, we conclude that the blob parameters obtained from the emission profile substantially reflect the parameters of the actual density blobs, although the density perturbation and emission perturbation are not identical. Figure 3 (a) also shows that the spatiotemporal pattern is almost point-symmetric with respect to its maximum. This implies that the above assumption, i.e., the blob does not change its shape and amplitude during propagation on the line of the diagnostic array for this blob event, is roughly satisfied in this case.

Using the proposed method, blob parameters are computed for each event. Distributions of $v, \theta, \delta x$ and $\delta I / \bar{I}$ as a function of $v_{x} \equiv v_{\text {peak }}$ are shown in Figs. 4 (b)-(e), respectively. In addition, Figs. 4 (a) and (f)-(i) depict histograms of $v_{x}, v, \theta$, $\delta x$ and $\delta I / \bar{I}$, respectively. Each histogram seems to have a certain central value and deviation, although the shape is not smooth because of the insufficient number of the ensemble. The value of $v_{x}$ scatters from $-100 \mathrm{~m} / \mathrm{s}$ to $400 \mathrm{~m} / \mathrm{s}$, where the central value is $\sim 200-300 \mathrm{~m} / \mathrm{s}$. The distribution of $v$ has a relatively slim shape with $v \sim 400 \pm 100 \mathrm{~m} / \mathrm{s}$. A peak of the propagation direction is found at $\theta \sim 45$ degree, with a positive tail of the distribution up to 90 degree. Linear regressions are also shown with blue thin lines. The speed of blob $v$ slightly correlates with $v_{x}$ whereas the correlation between $\theta$ and $v_{x}$ is much more clear. The correlation coefficients of $v$ and $\theta$ with respect to $v_{x}$ are 0.37 and -0.77 , respectively. This means that the variation of $v_{x}$ is mainly due to that of 
propagation direction $\theta$. A possible explanation of the observation is given as follows: In the SOL, a certain propagation velocity $v$ is determined by the local electric field inside the blob filament that is created by the dipole electrostatic potential structure. The blobs can travel in arbitrary directions depending on the orientation of the dipole vector, which determines the direction of the electric field in the blob filament and of the $E \times B$ direction. As a result, the radial propagation velocities $v_{x}$ strongly depends on the angles $\theta$ with almost fixed total blob velocity $v$. Figure $4(\mathrm{~d})$ shows that $v_{x}$ and $\delta x$ are almost independent (correlation coefficient between them is 0.085 ). Most of the evaluated width $\delta x$ is larger than the lower limit, which is determined by the cutoff scale of the spatial filter, and is distributed within $\delta x \sim 2-2.5 \pm 0.5 \mathrm{~cm}$. The blob size $a$ that corresponds to the observed $\delta x$ is $a \sim 1.5-2 \pm 1 \mathrm{~cm}$. These results are almost identical to what was evaluated by the traditional method, i.e., peak detection method, in the AUG experiment, where the comparison of the observations with scaling laws are given [12]. The typical emission amplitude is in the range of $\delta I / \bar{I} \sim 0.3 \pm 0.1$. Correlation between $\delta I / \bar{I}$ and $v_{x}$ is also unclear within the present number of ensembles.

Now, the influence of the frequency filter on the evaluated blob parameters is discussed. When a blob with the velocity of $v$ and the width of $2 a$ passes over the line of the detector array, the recorded time series at a detector forms a pulse width of $2 a / v$. The cutoff of the band-pass filter (lower cutoff $f>f_{\mathrm{c}, \mathrm{l}}=1 \mathrm{kHz}$ and higher cutoff $\left.f<f_{\mathrm{c}, \mathrm{h}}=10 \mathrm{kHz}\right)$ restricts the pulse width in time. If the pulse width $2 a / v$ is shorter than the inverse of the higher cutoff frequency $f_{\mathrm{c}, \mathrm{h}}^{-1}$, the higher frequency components of 
the signal are filtered out and the pulse is stretched up to the length $f_{\mathrm{c}, \mathrm{h}}^{-1}$. In this case, the evaluated relative blob amplitude $\delta n / n_{0}$ is decreased, although the peak position or time and evaluated blob size or velocity seems not to be disturbed. In the other case, when the pulse width $2 a / v$ is longer than the inverse of the lower cutoff frequency $f_{\mathrm{c}, \mathrm{l}}$, the signal is not detected as blob activity, but interpreted as a gradual change of the background density profile. Black dots in Fig. 5 exhibit the distribution of $v$ as a function of $2 \delta x$ in order to check the influence of the filter on the result. Most of the points are located within the two black solid lines, which show cutoff limits for low- and high-pass filter with the cutoff frequencies of $10 \mathrm{kHz}$ and $1 \mathrm{kHz}$, respectively. Note that several points distributed around the higher cutoff limitation is not owing to chopping off by the filter. Red dots in Fig. 5 indicate the same plot with another higher cutoff frequency of $f_{\mathrm{c}, \mathrm{h}}=15 \mathrm{kHz}$. Although individual locations of dots are not completely identical, the tendency of the distribution is almost unchanged. Little effect of the chopping off by filters is confirmed.

From Fig. 5, a correlation between $v$ and $\delta x$ ( $a$ also) is found. The regression line is calculated as $v=c_{0}+c_{1} \delta x$ where $c_{0}=3.6 \pm 98 \mathrm{~m} / \mathrm{s}$ and $c_{1}=190 \pm 441 / \mathrm{s}$, respectively, with a correlation coefficient of $\sim 0.64$. As shown in Fig. $4(\mathrm{~d})$, the correlation between $v_{x}$ and $\delta x$ was small. By taking into account the angle $\theta$ and looking at $v$, clear correlation between the blob velocity and size are found. In addition, the correlation coefficient between $v$ and $\theta$ is $\sim 0.15$, and a regression line is expressed as $v=c_{0}+c_{1} \theta$, where $c_{0}=340 \pm 83 \mathrm{~m} / \mathrm{s}$ and $c 1=1.1 \pm 1.5 \mathrm{~m} / \mathrm{s}$ degree. It is found that $v$ hardly correlates 
with $\theta$ in this discharge.

The validity of blob parameters evaluated with the new method is confirmed. In Fig. 1 (a), a blob signal is detected for the first time with a detector in the diagnostic array when $\overline{\mathrm{OB}}=a$. Then, the blob passes over the diagnostic array with $\overline{\mathrm{OB}}<a$, and finally, again $\overline{\mathrm{OB}}$ becomes $a$. The distance between two points where the blob is detected at the first time and last time is defined as $l_{\mathrm{tr}}$. From the trajectory of 'B' shown in Fig. $3(\mathrm{a}), l_{\mathrm{tr}}$ is evaluated. If the blob satisfies the three assumptions remarked above, a relation,

$$
\tan \theta=2 a / l_{\mathrm{tr}}
$$

should hold. Figure 6 examines Eq. (3) by plotting $2 \delta x / l_{\text {tr }}$ as a function of $\tan \theta$. Almost all points are distributed around the red dashed line showing Eq. (3), although a small offset, $2 \delta x / l_{\operatorname{tr}} \sim 0.5$, exists. This means that the assumptions made for the analysis essentially hold. Four dots having $\tan \theta>4$ correspond to the blob events with slower radial velocity $\left(\left|v_{x}\right|<100 \mathrm{~m} / \mathrm{s}\right)$ and larger angle $(\theta \sim 70-90$ degree $)$. For those blobs that propagate almost vertically through the detector array, $l_{\text {tr }}$ can be small value and thus becomes sensitive to noise. Therefore the large scatter of the values from Eq. (3) is seen.

\section{Conclusion and summary}

In this article, we have proposed a new method which evaluates the propagation angle, the velocity and the size of a plasma blob from one-dimensional data. The method 
calculates these quantities by means of two different velocities that are obtained from a one-dimensional measurement, i.e., the velocity of spatial- and temporal- peak positions in a spatiotemporal evolution signal. After the explanation of the calculation procedure, the method was applied to data from the Lithium beam emission spectroscopy (Li-BES) system in AUG. In the present experimental condition, the statistically evaluated blob parameters were: blob velocity of $v=400 \pm 100 \mathrm{~m} / \mathrm{s}$, blob radius of $a \sim 1.5-2 \pm 1 \mathrm{~cm}$ and propagation direction of $\theta \sim 40-90$ degree. Correlation between blob size and velocity of $\sim 0.64$ was clearly obtained, whereas that between blob size and radial velocity was less than 0.085. By employing a new degree of freedom, in this case the angle $\theta$, a new physical aspect was clarified.

Throughout the work, the capability of the Li-BES for the investigation of plasma blob dynamics is proved. In order to obtain more reliability of the new method, actual crosscheck with two-dimensional diagnostics is necessary.

\section{Acknowledgment}

The authors would like to thank the ASDEX Upgrade Team. One of the authors (T. K.) acknowledges professor A. Fujisawa, professor S. Sugita and professor Y. Kosuga for useful discussion. This work, supported by the European Commission under the Contract of Association between EURATOM and ÖAW, was carried out within the framework of the European Fusion Development Agreement (EFDA). In addition, this work is partly supported by the Grant-in-Aid for Scientific Research of JSPF (21224014, 
23244113), the Grant- in-Aid for JSPS Fellows (24-7632), the Educational and Research Promotion (Overseas Scientific Activity) Program 2012 for Doctor Course Students, offered by Interdisciplinary Graduate School of Engineering Sciences, Kyushu University. 


\section{Reference}

[1] K Itoh, S-I Itoh, and A Fukuyama. Transport and structural formation in plasmas. Institute of Physics Pub., 1999.

[2] D A D’Ippolito, J R Myra, and S I Krasheninnikov 2002 Phys. Plasmas 9 222-233

[3] S I Krasheninnikov, D A D’Ippolito, and J R Myra 102008 J. Plasma Phys. 74 679-717

[4] M V Umansky, S I Krasheninnikov, B LaBombard, and J L Terry 1998 Phys. Plasmas 53373

[5] M Kikuchi, S Nishio, G Kurita, K Tsuzuki, M Bakhtiari, H Kawashima, H Takenaga, Y Kusama, and K Tobita 2006 Fusion Eng. Des. 81 1589-1598

[6] S I Krasheninnikov 2001 Phys. Lett. A 283 368-370

[7] J R Myra and D A D'Ippolito 2005 Phys. Plasmas 12092511

[8] J R Myra, D A Russell, and D A D’Ippolito 2006 Phys. Plasmas 13112502

[9] J R Myra, D A D’Ippolito, D P Stotler, S J Zweben, B P LeBlanc, J E Menard, R J Maqueda, and J Boedo 2006 Phys. Plasmas 13092509

[10] C Theiler, I Furno, P Ricci, A Fasoli, B Labit, SH Müller, and G Plyushchev 2009 Phys. Rev. Lett. 103065001

[11] C Theiler, I Furno, A Fasoli, P Ricci, B Labit, and D Iraji 2011 Phys. Plasmas 18055901

[12] G Birkenmeier, F M Laggner, M Willensdorfer, T Kobayashi, P Manz, E Wolfrum, D Carralero, R Fischer, B Sieglin, G Fuchert, U Stroth, and the ASDEX Upgrade Team 2014 Plasma Phys. Control. Fusion 56075019

[13] P Manz, D Carralero, G Birkenmeier, H W Müller, S H Müller, G Fuchert, B D Scott, and U Stroth 2013 Phys. Plasmas 20102307

[14] S J Zweben, D P Stotler, J L Terry, B LaBombard, M Greenwald, M Muterspaugh, C S Pitcher, K Hallatschek, R J Maqueda, B Rogers, et al 2002 Phys. Plasmas 91981

[15] O Grulke, J L Terry, B LaBombard, and S J Zweben 2006 Phys. Plasmas 13012306

[16] J A Boedo, D L Rudakov, R A Moyer, G R McKee, R J Colchin, M J Schaffer, P G Stangeby, 
W P West, S L Allen, T E Evans, et al 2003 Phys. Plasmas 101670

[17] N Fedorczak, P Manz, S C Thakur, M Xu, G R Tynan, G S Xu, and S C Liu 2012 Phys. Plasmas 19 122302-122302

[18] G S Xu, Volker Naulin, W Fundamenski, C Hidalgo, J A Alonso, C Silva, B Goncalves, Anders Henry Nielsen, J Juul Rasmussen, S I Krasheninnikov, et al 2009 Nucl. Fusion 49 092002

[19] Min Xu, George Tynan, Patrick Diamond, Kaijun Zhao, Jun Cheng, Jiaqi Dong, Peter Manz, Nicolas Fedorczak, Saikat Thakur, Jonathan Yu, et al. Turbulent eddy-mediated transport in the edge of hl-2a tokamak plasma. In 24th IAEA Fusion Energy Conference (San Diego, 2012) IAEA CN-197/EX/7-2Rb. APS, 2012.

[20] M Willensdorfer, G Birkenmeier, R Fischer, FM Laggner, E Wolfrum, G Veres, F Aumayr, D Carralero, L Guimarãis, B Kurzan, et al 2014 Plasma Phys. Control. Fusion 56025008

[21] O E Garcia, N H Bian, and W Fundamenski 2006 Phys. Plasmas 13082309

[22] O E Garcia, J Horacek, R A Pitts, A H Nielsen, W Fundamenski, J P Graves, V Naulin, and J Juul Rasmussen 2006 Plasma Phys. Control. Fusion 48 L1

[23] Jens Madsen, Odd E Garcia, Jeppe Stærk Larsen, Volker Naulin, Anders H Nielsen, and Jens Juul Rasmussen 2011 Phys. Plasmas 18112504

[24] Ning Yan, Anders Henry Nielsen, G S Xu, Volker Naulin, J J Rasmussen, Jens Madsen, H Q Wang, S C Liu, W Zhang, L Wang, et al 2013 Plasma Phys. Control. Fusion 55115007

[25] B Kurzan and HD Murmann 2011 Rev. Sci. Instrum. 82 103501-103501

[26] M Willensdorfer, E Wolfrum, R Fischer, J Schweinzer, M Sertoli, B Sieglin, G Veres, F Aumayr, et al 2012 Rev. Sci. Instrum. 83 023501-023501

[27] Peter Bloomfield. Fourier analysis of time series: an introduction. Wiley. com, 2004. 
(a)

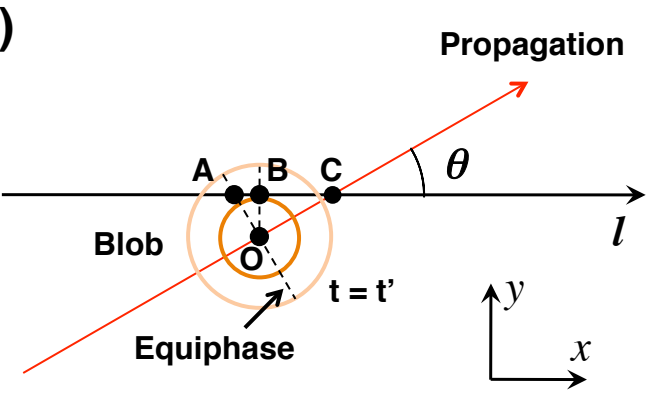

(b)

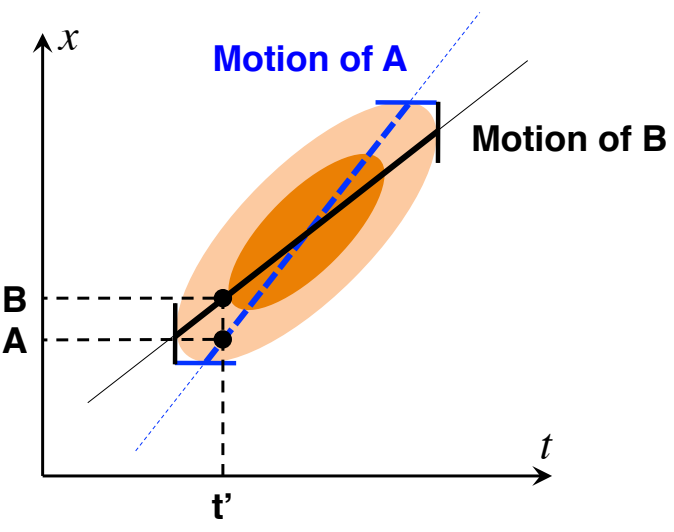

Figure 1. (a) Schematic view of the one-dimensional blob detection system and (b) a typical spatiotemporal evolution of the signal intensity. 


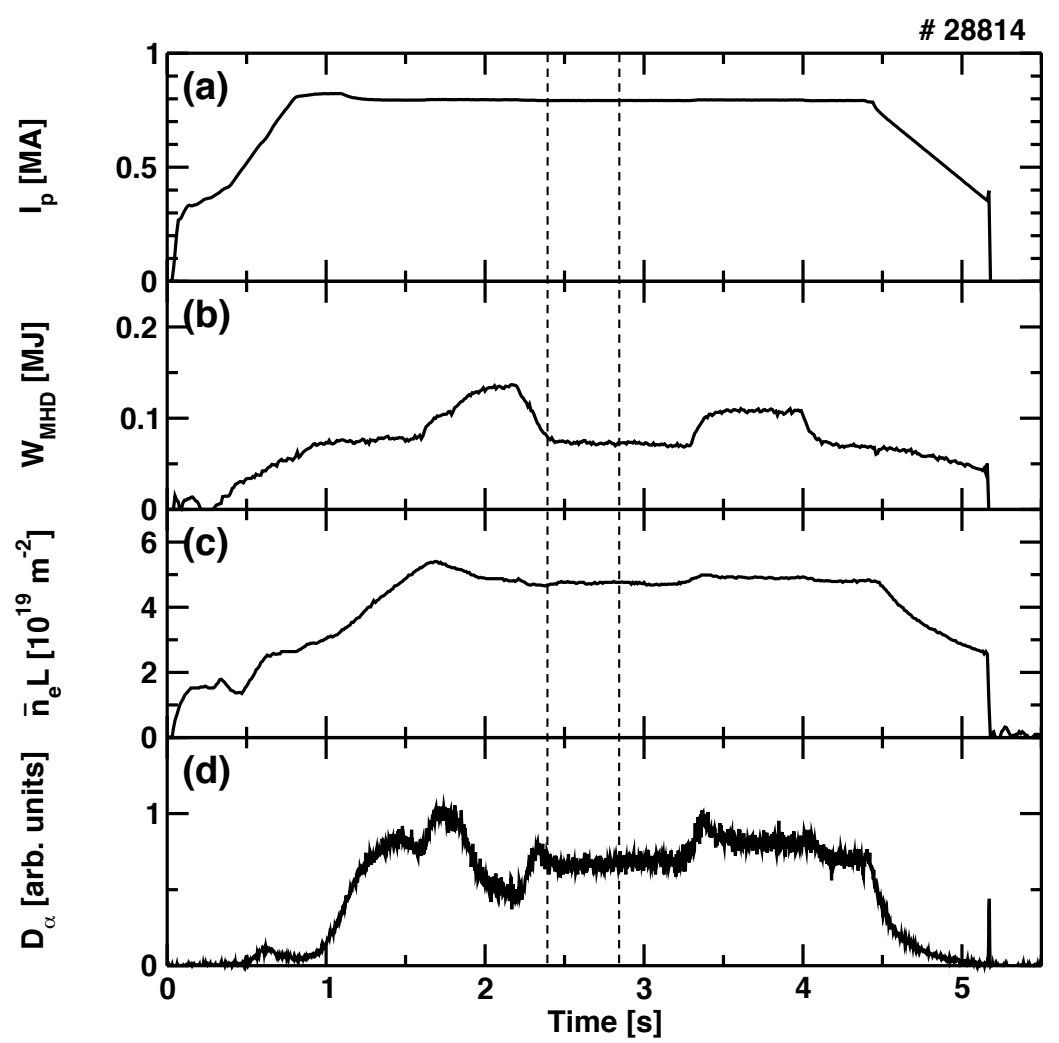

Figure 2. Time evolution of (a) plasma current $I_{\mathrm{p}}$, (b) plasma stored energy $W_{\mathrm{MHD}}$, (c) lineintegrated density with a line of sight through the plasma core region $\bar{n}_{\mathrm{e}} L$, and (d) $D_{\alpha}$ emission intensity. 

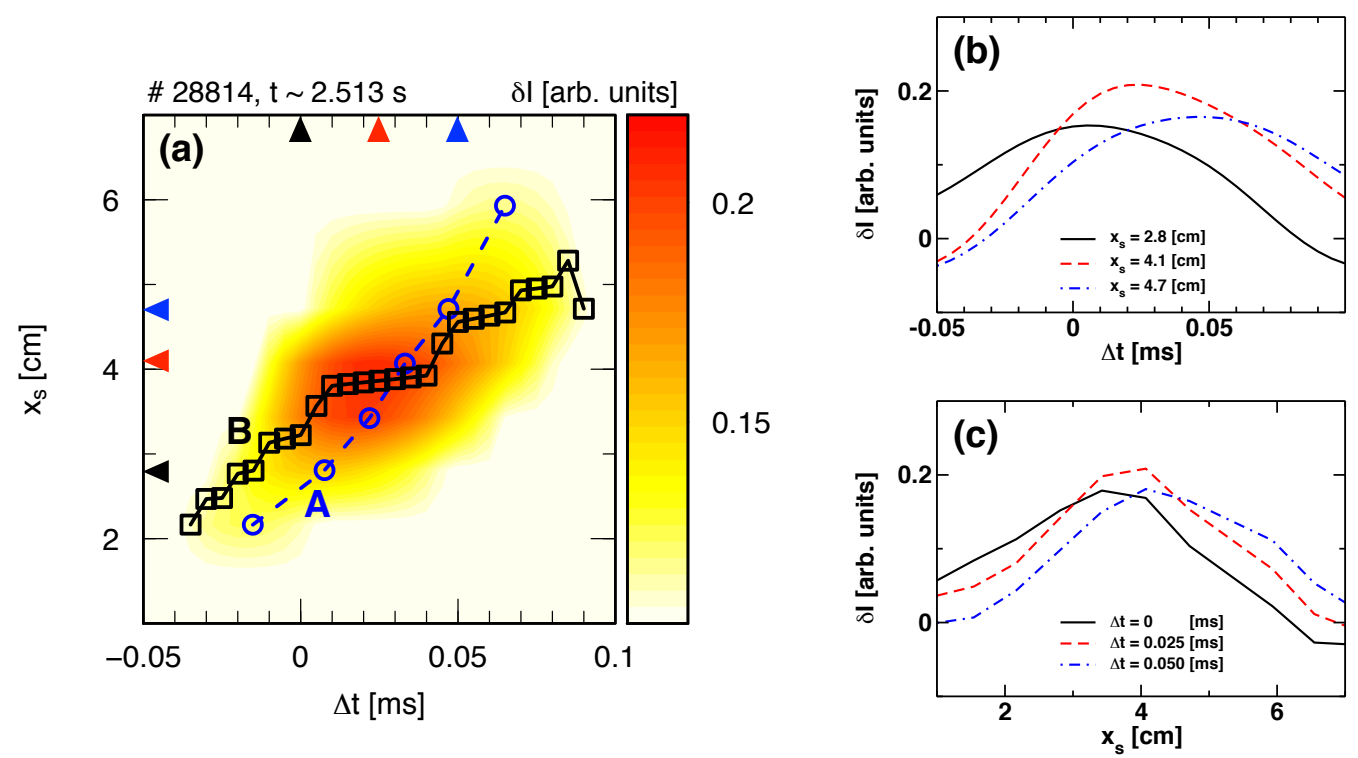

Figure 3. (a) Spatiotemporal evolution of a plasma blob at $t \sim 2.513 \mathrm{~s}$, and (b) its slices at the certain positions $\left(x_{\mathrm{s}}=2.8,4.1,4.7 \mathrm{~cm}\right)$ and $(\mathrm{c})$ those at the certain time points $(\Delta t=0,0.025,0.05 \mathrm{~ms})$. The positions and time points of the slices are depicted in the contour as triangles whose color correspond to that of the slice curve. Two curves in (a) indicate trajectories of 'A' and 'B', respectively. 


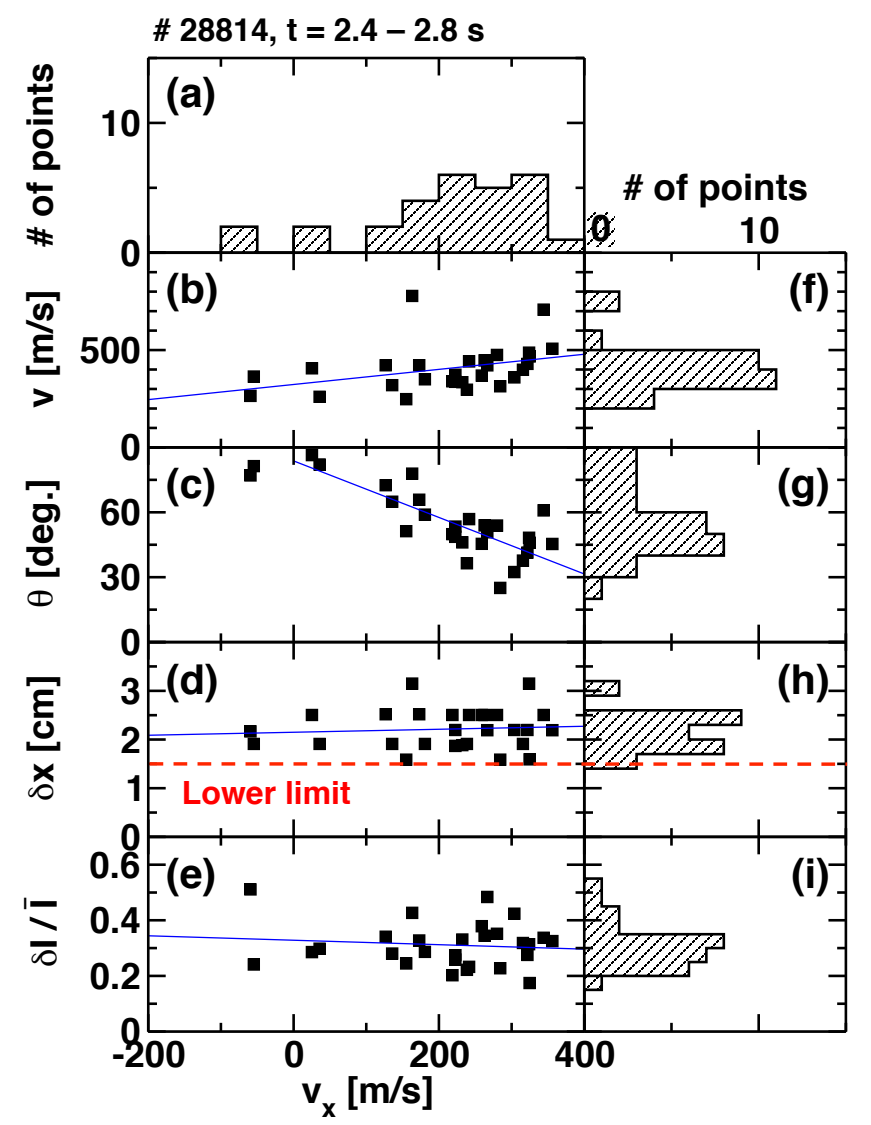

Figure 4. Estimated blob parameters. Distributions of $v, \theta, \delta x$ and $\delta I / \bar{I}$ as a function of $v_{x}$ with regression lines (blue thin lines) are shown in (b), (c), (d) and (e), respectively. (The regression line for (c) is calculated only for blobs with positive $v_{x}$ considering the symmetry in $\theta$. ) Histogram of (a) $v_{x}$, (f) $v$, (g) $\theta$, (h) $\delta x$ and (i) $\delta I / \bar{I}$ are also given. 


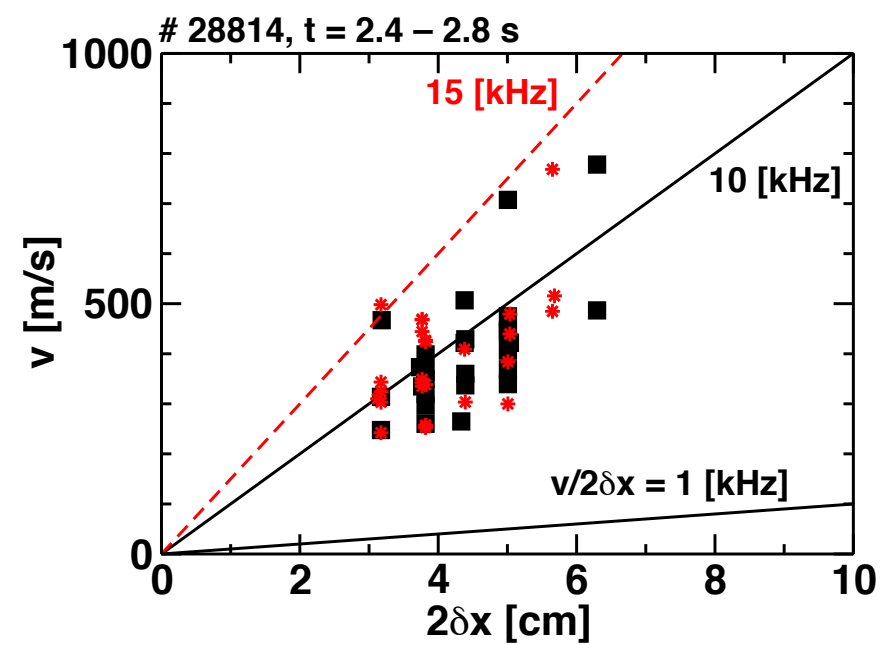

Figure 5. Distribution of $v$ as a function of $2 \delta x$. Black and red symbols exhibit the distributions calculated with two cutoff frequencies of the low-pass filter, $f_{\mathrm{c}, \mathrm{h}}=10 \mathrm{kHz}$ and $f_{\mathrm{c}, \mathrm{h}}=15 \mathrm{kHz}$, respectively. Two black solid lines indicate frequency filter limitations for the low- and the highpass filter with the cutoff frequencies of $10 \mathrm{kHz}$ and $1 \mathrm{kHz}$, respectively. Red dashed line shows that for the low-pass filter with the cutoff frequency of $15 \mathrm{kHz}$.

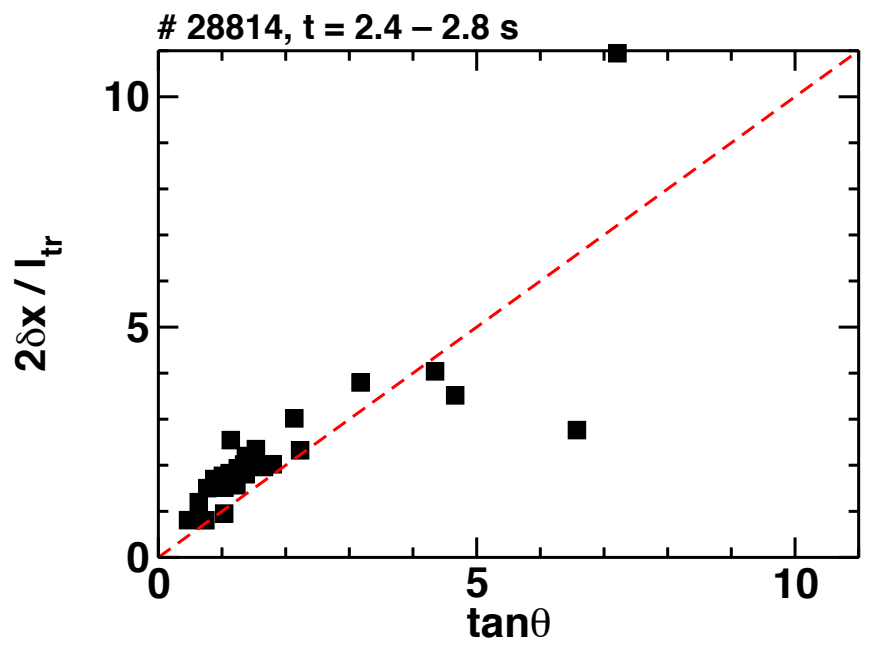

Figure 6. Distribution of $2 \delta x / l_{\operatorname{tr}}$ as a function of $\tan \theta$. Red dashed line indicates $2 \delta x / l_{\operatorname{tr}}=\tan \theta$ 